\title{
NORM ESTIMATES FOR FUNCTIONS OF TWO COMMUTING MATRICES*
}

\author{
MICHAEL GIL'†
}

\begin{abstract}
Matrix valued analytic functions of two commuting matrices are considered. A precise norm estimate is established. As a particular case, the matrix valued functions of two matrices on tensor products of Euclidean spaces are explored.
\end{abstract}

Key words. Functions of commuting matrices, Norm estimate.

AMS subject classifications. 15A15, 15A54, 15A69, 15A60.

1. Introduction and statement of the main result. In the book [4], I.M. Gel'fand and G.E. Shilov have established an estimate for the norm of a regular matrix-valued function in connection with their investigations of partial differential equations. However that estimate is not sharp, it is not attained for any matrix. The problem of obtaining a precise estimate for the norm of a matrix-valued function has been repeatedly discussed in the literature. In the paper [5] (see also [7]) the author has derived a precise estimate for a regular matrix-valued function. It is attained in the case of normal matrices. In the present paper we generalize the main result of the paper [5] to functions of two commuting matrices. Besides, the main result of the present paper-Theorem 1.1 is improved in the case of matrices on tensor products of Euclidean spaces.

It should be noted that functions of commuting operators were investigated by many mathematicians, cf. $[1,10,12]$ and references therein however the norm estimates were not considered, but as it is well-known, matrix valued functions are Green's functions and characteristic functions of various differential and difference equations. This fact allow us to investigate stability, well-posedness and perturbations of these equations by norm estimates for matrix valued functions, cf. $[2,3,6]$.

Let $\mathbb{C}^{n}$ be a Euclidean space with a scalar product $(\cdot, \cdot)$, the unit matrix $I$ and the Euclidean norm $\|\cdot\|=(\cdot, \cdot)^{1 / 2} ; M\left(\mathbb{C}^{n}\right)$ denotes the set of all linear operators in $\mathbb{C}^{n}$. For a $A \in M\left(\mathbb{C}^{n}\right),\|A\|$ is the operator norm; $N(A)$ is the Frobenius (Hilbert-Schmidt) norm: $N^{2}(A)=$ Trace $\left(A A^{*}\right) ; A^{*}$ is the operator adjoint to $A, \lambda_{j}(A), j=1, \ldots, n$ are the eigenvalues counting with their multiplicities, $\sigma(A)$ is the spectrum.

Everywhere below $A$ and $B$ are commuting matrices. Let $\Omega_{A}$ and $\Omega_{B}$ be open simple connected sets containing $\sigma(A)$ and $\sigma(B)$, respectively. Let $f$ be a scalar function analytic on $\Omega_{A} \times \Omega_{B}$. Introduce the operator valued function

$$
f(A, B):=-\frac{1}{4 \pi^{2}} \int_{L_{B}} \int_{L_{A}} f(z, w) R_{z}(A) R_{w}(B) d w d z,
$$

${ }^{*}$ Received by the editors 26 September 2004. Accepted for publication 6 March 2005. Handling Editor: Harm Bart.

${ }^{\dagger}$ Department of Mathematics, Ben Gurion University of the Negev, Beer-Sheva 84105, Israel (gilmi@cs.bgu.ac.il). 
where $L_{A} \subset \Omega_{A}, L_{B} \subset \Omega_{B}$ are closed contour surrounding $\sigma(A)$ and $\sigma(B)$, respectively. Note that if the series

$$
f(z, w)=\sum_{j, k=0}^{\infty} c_{j k} z^{j} w^{k}
$$

converges for $|z| \leq r_{s}(A),|w| \leq r_{s}(w)$, where $r_{s}(A)$ denotes the spectral radius of $A$, then (1.1) holds.

The following quantity plays a key role in the sequel

$$
g(A)=\left(N^{2}(A)-\sum_{k=1}^{n}\left|\lambda_{k}(A)\right|^{2}\right)^{1 / 2} .
$$

Since

$$
\sum_{k=1}^{n}\left|\lambda_{k}(A)\right|^{2} \geq \mid \text { Trace } A^{2} \mid \text {, we have } g^{2}(A) \leq N^{2}(A)-\mid \text { Trace } A^{2} \mid .
$$

If $A$ is a normal matrix, i.e. if $A A^{*}=A^{*} A$, then $g(A)=0$. Also the inequality

$$
g^{2}(A) \leq \frac{1}{2} N^{2}\left(A^{*}-A\right)
$$

is valid, cf. [7, Section 2.1]. Introduce the numbers

$$
\eta_{k}:=\frac{1}{k !} \sqrt{\frac{(n-1) !}{(n-k-1) !(n-1)^{k}}} \text { for } k=1, \ldots, n-1 ; \eta_{0}=1 .
$$

It is simple to check that

$$
\eta_{k}^{2} \leq \frac{1}{(k !)^{3}}(k=1, \ldots, n-1) .
$$

Denote by $c o(A), c o(B)$ the closed convex hulls of $\sigma(A)$ and $\sigma(B)$, respectively. Put

$$
f^{(j, k)}(z, w)=\frac{\partial^{j+k} f(z, w)}{\partial z^{j} \partial w^{k}} .
$$

Now we are in a position to formulate the main result of the paper.

THEOREM 1.1. Let $A$ and $B$ be commuting $n \times n$-matrices and $f(z, w)$ be regular on a neighborhood of $\operatorname{co}(A) \times \operatorname{co}(B)$. Then

$$
\|f(A, B)\| \leq \sum_{j, k=0}^{j+k \leq n-1} \eta_{j} \eta_{k} g^{j}(A) g^{k}(B) \sup _{z \in c o(A), w \in c o(B)}\left|f^{(j, k)}(z, w)\right| .
$$

The proof of this theorem is divided into a series of lemmas which are presented in the next section. If both $A$ and $B$ are normal operators, and

$$
\sup _{z \in c o(A), w \in c o(B)}|f(z, w)|=\sup _{z \in \sigma(A), w \in \sigma(B)}|f(z, w)|,
$$


then Theorem 1.1 gives us the exact relation

$$
\|f(A, B)\|=\sup _{z \in \sigma(A), w \in \sigma(B)}|f(z, w)| .
$$

Taking into account (1.3), we get

COROLlaRY 1.2. Under the hypothesis of Theorem 1.1, the estimate

$$
\|f(A, B)\| \leq \sum_{j, k=0}^{j+k \leq n-1} \frac{g^{j}(A) g^{k}(B)}{(j ! k !)^{3 / 2}} \sup _{z \in c o(A), w \in c o(B)}\left|f^{(j, k)}(z, w)\right|
$$

is true.

Let $A$ be a normal matrix. Then $g(A)=0$. Now Corollary 1.2 implies

$$
\|f(A, B)\| \leq \sum_{0 \leq k \leq n-1} \frac{g^{k}(B)}{(k !)^{3 / 2}} \sup _{z \in c o(A), w \in c o(B)}\left|\frac{\partial^{k} f(z, w)}{\partial w^{k}}\right| .
$$

Let us evaluate the error of Theorem 1.1 in the case of non-normal matrices. Certainly, we can obtain the exact value of the norm of a function of two matrices in very simple cases only. Consider the $2 \times 2$-matrices

$$
A=\left(\begin{array}{cc}
a & 1 / 3 \\
0 & a
\end{array}\right)
$$

and $B=2 A$ with $a>0$. Construct a function of two variables by setting $(x, y) \rightarrow$ $f(x+y)$. Direct calculations show that

$$
f(A+B)=\left(\begin{array}{cc}
f(3 a) & f^{\prime}(3 a) \\
0 & f(3 a)
\end{array}\right)
$$

Assume that $f(3 a)=0$. Then $\|f(A+B)\|=\left|f^{\prime}(3 a)\right|$. At the same time, Theorem 1.1 gives us the relations,

$$
\|f(A+B)\| \leq|f(a)|+\left|f^{\prime}(3 a)\right|(g(A)+g(B))=\left|f^{\prime}(3 a)\right|,
$$

since $g(A)=1 / 3, g(B)=2 / 3$. Thus in the considered case Theorem 1.1 gives us the exact result.

EXAmple 1.3. Consider the polynomial

$$
P(z, w)=\sum_{\nu=0}^{m_{1}} \sum_{l=0}^{m_{2}} c_{\nu l} z^{\nu} w^{l}
$$

with complex, in general, coefficients. Then

$$
P^{(j, k)}(z, w)=\sum_{\nu=0}^{m_{1}-j} \sum_{l=0}^{m_{2}-k} c_{\nu l} \nu(\nu-1) \ldots(\nu-j+1) z^{\nu-j} l \ldots(l-k+1) w^{l-k} .
$$


Now Theorem 1.1 implies

$$
\|P(A, B)\| \leq \sum_{j, k=0}^{j+k \leq n-1} \eta_{j} \eta_{k} g^{j}(A) g^{k}(B) \sum_{\nu=0}^{m_{1}-j} \sum_{l=0}^{m_{2}-k} \frac{\nu ! l !\left|c_{\nu l}\right|}{(l-k) !(\nu-j) !} r_{s}^{\nu-j}(A) r_{s}^{l-k}(B) .
$$

Recall that $r_{s}(.$,$) denotes the spectral radius. If both A$ and $B$ are normal operators, then

$$
\|P(A, B)\| \leq \sum_{\nu=0}^{m_{1}} \sum_{l=0}^{m_{2}}\left|c_{\nu l}\right| r_{s}^{\nu}(A) r_{s}^{l}(B)
$$

EXAMPLE 1.4. Consider the function

$$
f(z, w)=\cos (x z+y w) \quad(y, x \geq 0) .
$$

Note that the function $U(x, y)=\cos (x A+y B)$ is a solution of the equation

$$
\partial^{2} U(x, y) / \partial x^{2}+\partial^{2} U(x, y) / \partial y^{2}+\left(A^{2}+B^{2}\right) U(x, y)=0 .
$$

In the considered case

$$
\begin{aligned}
& \left|f^{(j, k)}(z, w)\right|=x^{j} y^{k}|\cos (x z+y w)|(j+k \text { is even }), \\
& \left|f^{(j, k)}(z, w)\right|=x^{j} y^{k}|\sin (x z+y w)|(j+k \text { is odd }) .
\end{aligned}
$$

For simplicity assume that the spectra of both $A$ and $B$ are real. Then thanks to Theorem 1.1,

$$
\|U(x, y)\| \leq \sum_{j, k=0}^{j+k \leq n-1} \eta_{j} \eta_{k} g^{j}(A) g^{k}(B) x^{j} y^{k} \quad(x, y \geq 0) .
$$

2. Proof of Theorem 1.1. The following lemma is needed.

LEMma 2.1. Let $\Omega$ and $\tilde{\Omega}$ be the closed convex hulls of complex, in general, points

$$
x_{0}, x_{1}, \ldots, x_{n}
$$

and

$$
y_{0}, y_{1}, \ldots, y_{m}
$$

respectively, and let a scalar-valued function $f(z, w)$ be regular on $D \times \tilde{D}$, where $D$ and $\tilde{D}$ are neighborhoods of $\Omega$ and $\tilde{\Omega}$, respectively. In addition, let $L \subset D, \tilde{L} \subset \tilde{D}$ be Jordan closed contours surrounding the points in (2.1) and (2.2), respectively. Then with the notation

$$
Y\left(x_{0}, \ldots, x_{n} ; y_{0}, \ldots y_{m}\right)=-\frac{1}{4 \pi^{2}} \int_{L} \int_{\tilde{L}} \frac{f(z, w) d z d w}{\left(z-x_{0}\right) \cdots\left(z-x_{n}\right)\left(w-y_{0}\right) \cdots\left(w-y_{m}\right)},
$$


we have

$$
\left|Y\left(x_{0}, \ldots, x_{n} ; y_{0}, \ldots y_{m}\right)\right| \leq \frac{1}{n ! m !} \sup _{z \in \Omega, w \in \tilde{\Omega}}\left|f^{(n, m)}(z, w)\right|
$$

Proof. First, let all the points be distinct: $x_{j} \neq x_{k}, y_{j} \neq y_{k}$ for $j \neq k$. Let a function $h$ of one variable be regular on $D$ and $\left[x_{0}, x_{1}, \ldots, x_{n}\right] h$ be a divided difference of function $h$ at points $x_{0}, x_{1}, \ldots, x_{n}$. Then

$$
\left[x_{0}, x_{1}, \ldots, x_{n}\right] h=\frac{1}{2 \pi i} \int_{L} \frac{h(\lambda) d \lambda}{\left(\lambda-x_{0}\right) \cdots\left(\lambda-x_{n}\right)}
$$

(see [4, formula (54)]). Thus

$$
Y\left(x_{0}, \ldots x_{n} ; y_{0}, \ldots, y_{m}\right)=\frac{1}{2 \pi i} \int_{\tilde{L}} \frac{\left[x_{0}, \ldots, x_{n}\right] f(., w) d w}{\left(w-y_{0}\right) \cdots\left(w-y_{n}\right)} .
$$

Now apply $(2.3)$ to $\left[x_{0}, \ldots x_{n}\right] f(., w)$. Then

$$
Y\left(x_{0}, \ldots x_{n} ; y_{0}, \ldots, y_{m}\right)=\left[x_{0}, \ldots x_{n}\right]\left[y_{0}, \ldots y_{m}\right] f(., .) \equiv\left[x_{0}, \ldots x_{n}\right]\left(\left[y_{0}, \ldots y_{m}\right] f(., .)\right) .
$$

The following estimate is well-known $[11$, p. 6$]$ :

$$
\left|\left[x_{0}, \ldots x_{n}\right]\left[y_{0}, \ldots y_{m}\right] f(., .)\right| \leq \sup _{z \in \Omega, w \in \tilde{\Omega}}\left|f^{(n, m)}(z, w)\right| .
$$

It proves the required result if all the points are distinct. If some points coincide, then the claimed inequality can be obtained by small perturbations and the previous arguments.

Furthermore, since $A$ and $B$ commute they have the same orthogonal normal basis of the triangular representation (Schur's basis) $\left\{e_{k}\right\}$. We can write

$$
A=D_{A}+V_{A}, B=D_{B}+V_{B},
$$

where $D_{A}, D_{B}$ are the diagonal parts, $V_{A}$ and $V_{B}$ are the nilpotent parts of $A$ and $B$, respectively. Furthermore, let $\left|V_{A}\right|$ be the operator whose matrix elements in $\left\{e_{k}\right\}$ are the absolute values of the matrix elements of the nilpotent part $V_{A}$ of $A$ with respect to this basis. That is,

$$
\left|V_{A}\right|=\sum_{k=1}^{n} \sum_{j=1}^{k-1}\left|a_{j k}\right|\left(., e_{k}\right) e_{j}
$$

where $a_{j k}=\left(A e_{k}, e_{j}\right)$. Similarly $\left|V_{B}\right|$ is defined.

LEMMA 2.2. Under the hypothesis of Theorem 1.1 the estimate

$$
\|f(A, B)\| \leq \sum_{j, k=0}^{j+k \leq n-1} \sup _{z \in c o(A), w \in \operatorname{co}(B)}\left|f^{(j, k)}(z, w)\right| \frac{\left\|\left|V_{A}\right|^{j}\left|V_{B}\right|^{k}\right\|}{j ! k !}
$$


is true, where $V_{A}$ and $V_{B}$ are the nilpotent part of $A$ and $B$, respectively.

Proof. It is not hard to see that the representation (2.4) implies the equality

$$
(A-I \lambda)^{-1}=\left(D_{A}+V_{A}-\lambda I\right)^{-1}=\left(I+R_{\lambda}\left(D_{A}\right) V_{A}\right)^{-1} R_{\lambda}\left(D_{A}\right)
$$

for all regular $\lambda$. According to Lemma 1.7.1 from [7] $R_{\lambda}\left(D_{A}\right) V_{A}$ is a nilpotent operator because $V$ and $R_{\lambda}\left(D_{A}\right)$ the same invariant subspaces. Hence, $\left(R_{\lambda}\left(D_{A}\right) V_{A}\right)^{n}=0$. Therefore,

$$
R_{z}(A)=\sum_{k=0}^{n-1}\left(R_{z}\left(D_{A}\right) V_{A}\right)^{k}(-1)^{k} R_{z}\left(D_{A}\right)
$$

Similarly,

$$
R_{\mu}(B)=\sum_{k=0}^{n-1}\left(R_{\mu}\left(D_{B}\right) V_{B}\right)^{k}(-1)^{k} R_{\mu}\left(D_{B}\right)
$$

So from (1.1) it follows

$$
f(A, B)=\sum_{j, k=0}^{n-1} C_{j k}
$$

where

$$
C_{j k}=\frac{(-1)^{k+j}}{4 \pi^{2}} \int_{L_{B}} \int_{L_{A}} f(z, w)\left(R_{z}\left(D_{A}\right) V_{A}\right)^{j} R_{z}\left(D_{A}\right)\left(R_{w}\left(D_{B}\right) V_{B}\right)^{k} R_{w}\left(D_{B}\right) d z d w .
$$

Since $D_{A}$ is a diagonal matrix with respect to the Schur basis $\left\{e_{k}\right\}$ and its diagonal entries are the eigenvalues of $A$, then

$$
R_{z}\left(D_{A}\right)=\sum_{j=1}^{n} \frac{Q_{j}}{\lambda_{j}(A)-z}
$$

where $Q_{k}=\left(., e_{k}\right) e_{k}$. Similarly,

$$
R_{z}\left(D_{B}\right)=\sum_{j=1}^{n} \frac{Q_{j}}{\lambda_{j}(B)-z} .
$$

Taking into account that $Q_{s} V_{A} Q_{m}=0, Q_{s} V_{B} Q_{m}=0(s \geq m)$, we get

$$
\begin{gathered}
C_{j k}=\sum_{1 \leq s_{1}<s_{2}<\ldots<s_{j+1}<m_{1}<m_{2}<\ldots<m_{k+1}<n} Q_{s_{1}} V_{A} Q_{s_{2}} V_{A} \ldots \\
V_{A} Q_{s_{j+1}} Q_{m_{1}} V_{B} Q_{m_{2}} V_{B} \ldots V_{B} Q_{m_{k+1}} J\left(s_{1}, \ldots, s_{j+1}, m_{1}, \ldots, m_{k+1}\right),
\end{gathered}
$$


where

$$
\begin{gathered}
J\left(s_{1}, \ldots, s_{j+1}, m_{1}, \ldots m_{k+1}\right)= \\
\frac{(-1)^{k+j}}{4 \pi^{2}} \int_{L_{A}} \int_{L_{B}} \frac{f(z, w) d z d w}{\left(\lambda_{s_{1}}(A)-z\right) \cdots\left(\lambda_{s_{k+1}}(A)-z\right)\left(\lambda_{m_{1}}(B)-w\right) \cdots\left(\lambda_{m_{k+1}}(B)-w\right)} .
\end{gathered}
$$

Lemma 2.5.1 from [7] gives us the estimate

$$
\left\|C_{j k}\right\| \leq \max _{1 \leq s_{1}<\ldots<s_{j+1}<m_{1}<\ldots<m_{k+1}<n}\left|J\left(s_{1}, \ldots, s_{j+1}, m_{1}, \ldots m_{k+1}\right)\right|\left\|\left|V_{A}\right|^{j}\left|V_{B}\right|^{k}\right\| .
$$

Due to Lemma 2.1,

$$
\left|J\left(s_{1}, \ldots, s_{j+1}, m_{1}, \ldots, m_{k+1}\right)\right| \leq \sup _{z \in c o(A), w \in c o(B)} \frac{\left|f^{(j, k)}(z, w)\right|}{j ! k !} .
$$

This inequality and (2.5) imply the required result. $\square$

Proof of Theorem 1.1: Theorem 2.5.1 from [7] implies

$$
\left\|V^{k}\right\| \leq \eta_{k} k ! N^{k}(V)
$$

for any nilpotent matrix $V \in M\left(\mathbb{C}^{n}\right)$. Take into account that $N\left(\left|V_{A}\right|\right)=N\left(V_{A}\right)$. Moreover, thanks to Lemma 2.3.2 from [7], $N\left(V_{A}\right)=g(A)$. Thus

$$
\left\|\left|V_{A}\right|^{k}\right\| \leq k ! \eta_{k} g^{k}(A)(k=1, \ldots, n-1)
$$

The similar inequality holds for $V_{B}$. Now the previous lemma yields the required result.

3. Functions of matrices on tensor products. Let $E_{1}=\mathbb{C}^{n_{1}}, E_{2}=\mathbb{C}^{n_{2}}$, be the Euclidean spaces of the dimensions $n_{1}$ and $n_{2}$, with the scalar products $<., .>_{1}$ and $<., .>_{2}$, respectively, and the norms $\|\cdot\|_{l}=\sqrt{<., .>_{l}}(l=1,2)$. Let $H=E_{1} \otimes E_{2}$ be the tensor product of $E_{1}$ and $E_{2}$ with the scalar product defined by

$$
<y \otimes h, y_{1} \otimes h_{1}>_{H} \equiv<y, y_{1}>_{1}<h, h_{1}>_{2} \quad\left(y, y_{1} \in E_{1} ; h, h_{1} \in E_{2}\right)
$$

and the cross norm $\|\cdot\|_{H}=\sqrt{<., \cdot\rangle_{H}}$, cf. [9]. In addition, $I=I_{H}$ and $I_{l}$ mean the unit operators in $H$ and $E_{l}$, respectively. So $H=\mathbb{C}^{n}$ with $n=n_{1} n_{2}$.

Recall that $M(E)$ is the set of all linear operators in a space $E$. In this section it is assumed that $A \in M\left(E_{1}\right)$ and $B \in M\left(E_{2}\right)$.

Again let $\Omega_{A}$ and $\Omega_{B}$ be open simple connected sets containing $\sigma(A)$ and $\sigma(B)$, respectively. Let $f$ be a scalar function analytic on $\Omega_{A} \times \Omega_{B}$. Introduce the operator valued function

$$
f(A, B):=-\frac{1}{4 \pi^{2}} \int_{L_{B}} \int_{L_{A}} f(z, w) R_{z}(A) \otimes R_{w}(B) d w d z
$$


where $L_{A} \subset \Omega_{A}, L_{B} \subset \Omega_{B}$ are closed contour surrounding $\sigma(A)$ and $\sigma(B)$, respectively. If the series

$$
f(z, w)=\sum_{k=0}^{\infty} \sum_{j=0}^{\infty} c_{j k} z^{j} w^{k}
$$

converges for $|z| \leq r_{s}(A),|w| \leq r_{s}(B)$, where $r_{s}(A)$ is the spectral radius of $A$, then (3.1) holds. Besides,

$$
f(z, w)=\sum_{k=0}^{\infty} \sum_{j=0}^{\infty} c_{j k} A^{j} \otimes B^{k}
$$

Theorem 3.1. Let $A \in M\left(E_{1}\right)$ and $B \in M\left(E_{2}\right)$ and $f(z, w)$ be regular on a neighborhood of $\operatorname{co}(A) \times \operatorname{co}(B)$. Then

$$
\|f(A, B)\|_{H} \leq \sum_{j=0}^{n_{1}-1} \sum_{k=0}^{n_{2}-1} \eta_{j} \eta_{k} g^{j}(A) g^{k}(B) \sup _{z \in c o(A), w \in c o(B)}\left|f^{(j, k)}(z, w)\right| .
$$

Proof. Put $\tilde{A}=A \otimes I_{2}, \tilde{B}=I_{1} \otimes B_{2}$. Now Lemma 2.2 implies

$$
\|f(A, B)\|_{H} \leq \sum_{j, k=0}^{j+k \leq n-1} \sup _{z \in c o(A), w \in c o(B)}\left|f^{(j, k)}(z, w)\right| \frac{\left\|\left|V_{\tilde{A}}\right|^{j}\left|V_{\tilde{B}}\right|^{k}\right\|_{H}}{j ! k !}
$$

where $V_{\tilde{A}}, V_{\tilde{B}}$ are the nilpotent parts of $\tilde{A}$ and $\tilde{B}$, respectively. But

$$
V_{\tilde{A}}^{n_{1}}=V_{A}^{n_{1}} \otimes I_{2}=0 .
$$

Similarly, $V_{\widetilde{B}}^{n_{2}}=0$. Thus,

$$
\|f(A, B)\|_{H} \leq \sum_{k=0}^{n_{2}} \sum_{j=0}^{n_{1}} \sup _{z \in c o(A), w \in c o(B)}\left|f^{(j, k)}(z, w)\right| \frac{\left\|\left|V_{A}\right|^{j}\right\|_{1}\left\|\left|V_{B}\right|^{k}\right\|_{2}}{j ! k !}
$$

Now the required result follows from (2.7).

Taking into account (1.3), we get

Corollary 3.2. Under the hypothesis of Theorem 3.1, the estimate

$$
\|f(A, B)\|_{H} \leq \sum_{k=0}^{n_{2}-1} \sum_{j=0}^{n_{1}-1} \frac{g^{j}(A) g^{k}(B)}{(j ! k !)^{3 / 2}} \sup _{z \in c o(A), w \in c o(B)}\left|f^{(j, k)}(z, w)\right|
$$

is true. 
Acknowledgment. I am very grateful to the referee for very helpful remarks.

\section{REFERENCES}

[1] R. Arens and A.P. Calderon. Analytic functions of several Banach algebra elements. Ann. Math., 62:204-216, 1955.

[2] Yu L. Daleckii and M.G. Krein. Stability of Solutions of Differential Equations in Banach Space. Amer. Math. Soc., Providence, R.I., 1974.

[3] M.I. Gel'fand and G.E. Shilov. Some Questions of Theory of Differential Equations. Nauka, Moscow (in Russian), 1958.

[4] A.O. Gel'fond. Calculations of Finite Differences. Nauka, Moscow (in Russian), 1967.

[5] M.I. Gil'. Estimates for norm of matrix-valued functions, Linear and Multilinear Algebra, 35:65$73,1993$.

[6] M.I. Gil'. Stability of Finite and Infinite Dimensional Systems. Kluwer Academic Publishers, Boston-Dordrecht-London, 1998.

[7] M.I. Gil'. Operator Functions and Localization of Spectra. Lectures Notes In Mathematics vol. 1830, Springer-Verlag, Berlin, 2003.

[8] R.A. Horn and C.R. Johnson. Topics in Matrix Analysis. Cambridge University Press, Cambridge, 1991.

[9] M. Marcus. Finite Dimensional Multilinear Algebra, Vol. I, Marcel Dekker, New York, 1973.

[10] V. Müller. Spectral Theory of Linear Operators. Birkhäusr Verlag, Basel, 2003.

[11] A.M. Ostrowski. Solution of Equations in Euclidean and Banach spaces. Academic Press, New York - London, 1973.

[12] J.L. Taylor. Analytic functional calculus for several commuting operators. Acta Math., 125:138, 1970. 